\title{
Analysis of cosmic-ray interaction in balloon-borne emulsion detector
}

Hiroaki Kawahara* On behalf of GRAINE collaboration

Nagoya University, J-464-8602, Japan

E-mail: kawahara@flab.phys.nagoya-u.ac.jp

\begin{abstract}
GRAINE (Gamma-Ray Astro-Imager with Nuclear Emulsion) is a project aiming to observe cosmic gamma-ray sources. The main detector is consists of emulsion films and have sub-micron accuracy for tracking electron pairs from gamma ray interactions. In 2015, we succeeded 14 hour balloon flight in Australia Alice-Springs, and analysis of millions of cosmic-ray and gamma-ray events is undergoing.

We analyzed hadronic interaction in the emulsion chamber to evaluate the performance of the telescope. I detected about $1.5 \times 10^{5}$ hadronic events in one module and identified gamma rays attaching the events. As a result, we proved that angular resolution is about five times superior to existing telescopes.
\end{abstract}

The 3rd International Symposium on "Quest for the Origin of Particles and the Universe" 5-7 January 2017

Nagoya University, Japan

${ }^{*}$ Speaker. 


\section{Introduction}

\subsection{Gamma-ray astronomy}

Origin of high energy cosmic rays is an important subject even now. Supernova, pulser, AGN, GRB and other high energy phenomena in the universe are the candidates of the origin of cosmic rays. One of the most suitable probe for research of these is gamma ray. Gamma rays are emitted from cosmic rays via synchrotron radiation, inverse Compton scattering or creation of pi-zero particle. Because gamma rays are not affected by interstellar magnetic field, gamma rays can give us good information about origin of cosmic rays.

\subsection{Present situation}

Fermi satellite was launched by NASA in 2008. It has a gamma-ray telescope called Large Area Telescope (Fermi-LAT). Fermi-LAT observes from sub-GeV to $100 \mathrm{GeV}$ energy region and detected over 3,000 objects in 4 years observation [1]. Improvement of angular resolution is essential for the post-Fermi telescopes. Resolution of $5 \mathrm{deg}$. at $100 \mathrm{MeV}$ and $0.8 \mathrm{deg}$. at $1 \mathrm{GeV}$ is the performance of Fermi-LAT. This is worse than other wave length observation such as X-rays, visible lights, micro-waves, radio-waves. We can expect progress of gamma ray astronomy by improvement of angular resolution of telescope.

\section{GRAINE project}

\subsection{Overview}

GRAINE is a project aiming to observe cosmic gamma rays using nuclear emulsion. The gamma-ray detector consists of stack of films. Structure of the films is that $65 \mu \mathrm{m}$ thick emulsion layers are applied on both sides of $180 \mu \mathrm{m}$ thick polystyrene base. 100 of films are piled up for an active converter that has 0.55 radiation length. Gamma rays react electron-pair creation in the converter. We measure the track of electrons and define the incident angle of gamma rays. Emulsion multi-stage shifter [2] are placed at downstream of the converter to get incident timing of gamma rays and star tracking cameras are used for attitude monitor.

\subsection{Performance and progress}

Fig 1 shows expected angular resolution of emulsion telescope. The solid line is indicate the performance estimated by Monte Carlo simulation. Some points with error bars are experimental result. We performed balloon experiments in 2011 in Japan and in 2015 Australia. $10 \mathrm{~m}^{2}$ aperture area telescope and long duration time flight realize accurate imaging of SNRs and measurement of spectrum and measurement of polarization of pulser.

\subsection{Balloon fight in 2015 in Australia}

The 2nd balloon fight of GRAINE was performed in 2015 in Alice Springs, Australia[3]. The target of the flight is detection of Vela pulsar which is known as the brightest gamma-ray source in $100 \mathrm{MeV}$ region. Fig 2 shows a photo of the telescope used for the flight. The telescope consists of 4 modules and aperture area is $3880 \mathrm{~cm}^{2}$. The telescope was launched at 6:33 (NT) on 12th of May 
and landed on the ground at 20:52. We succeeded to get 11.5 hours of observation time at altitude over $35 \mathrm{~km}$ including 6.5 hours of observation time for Vela pulsar. Scanning of emulsion film was carried out at Nagoya University in Japan by using Scanning machine called Hyper Track Selector (HTS). Detail of the system is introduced in the reference [4].

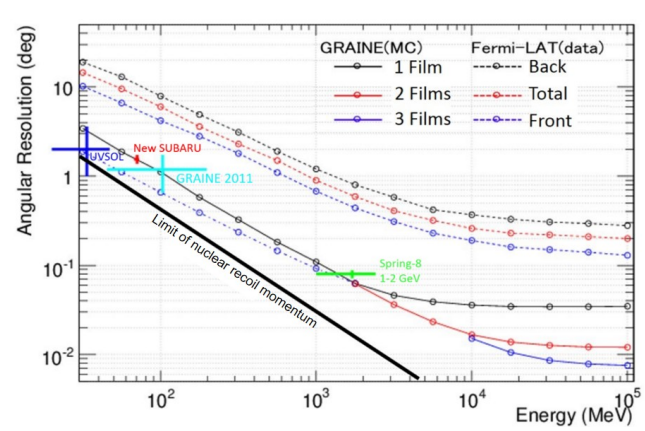

Figure 1: Angular resolution of the emulsion telescope by Monte Carlo simulation.

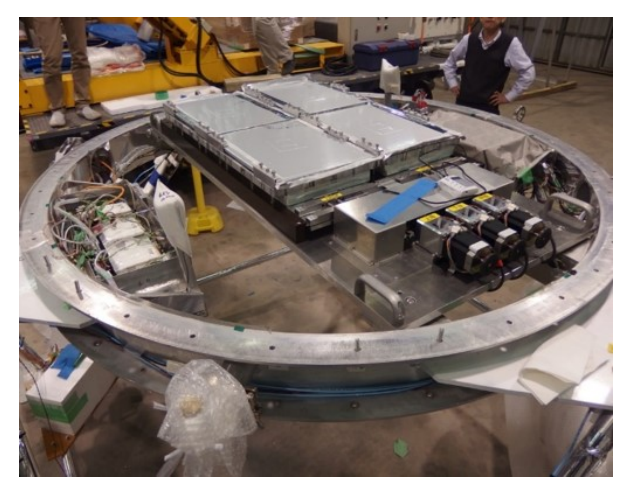

Figure 2: An emulsion gamma-ray telescope mounted on the gondra of GRAINE2015.

\section{Analysis of hadronic interaction}

\subsection{Motivation for the analysis}

Billions of cosmic-rays are recorded in emulsion films. The main component of them is cosmic-ray protons. Protons react with nuclei by the strong interaction, and the reaction is called hadronic interaction. Baryons and mesons are emitted from hadronic interaction, and gamma rays are also emitted from decay of pi-zero mesons. We conceived that these gamma rays are useful for evaluation of angular resolution and calibration of the detector.

\subsection{Interaction search}

Hadronic interactions are found as vertex point of tracks in emulsion.To search vertexes from large volume, we tried the following method.

- Defining target film and target volume

We will search vertex point in target volume that defined $0 \sim 600 \mu \mathrm{m}$ from the target film.

- Excluding penetrating tracks

To exclude tracks which can be found in upward of target volume, which means the tracks are passed the volume without hadronic interaction.

- Excluding disconnecting tracks

To exclude tracks which cannot be found in downward of target film, which means the tracks have low energy $(<100 \mathrm{MeV})$ or the tracks passed the film before construction of the chamber.

- Searching vertex points

By using remaining tracks, vertex points are searched in the target volume. 
- Repeat this process all over the volume of the emulsion chamber.

Fig 3 shows an example of hadronic interaction found by the method. The upper-left figure is the view from top of the detector. The blue segments indicate the tracks on target plate, and the red segments indicate the tracks found in upper-side of the vertex. The upper-right and the bottom-left figure is the view from side of the detector. Fig 4 shows the multiplicity distribution of the found event. 155 thousand of events are detected in a module. We checked the events by 3D-viewer and evaluated that reliability is over $90 \%$ in events that have five or more multiplicity, which means that they are not chance-coincidence vertexes.
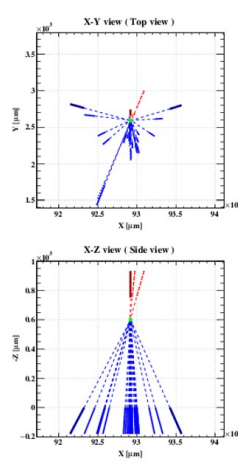

Figure 3: A sample of hadronic event. Reconstructed image and IP distribution.
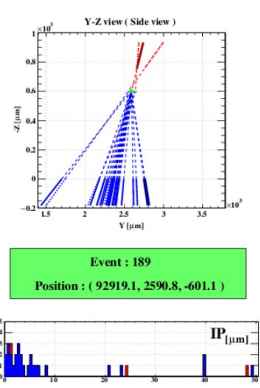

Figure 4: Multiplicity distribution of detected event in one chamber (the 3rd module).

\subsection{Linkage between hadronic interactions and gamma-rays}

To identify hadronic events that emitted gamma rays, we used timing information from emulsion multi-stage shifter, because it was difficult to identify two event several $\mathrm{cm}$ away without timing information. We connected the tracks to the bottom of the chamber and analyzed incident timing to the shifter. By these processes, we succeeded to select pairs of hadronic event and gammaray event which have same timing information. The time window was $\sim 18$ minute, because only first stage of the shifter was used for this analysis.

\section{Evaluation of angular resolution of gamma rays}

\subsection{Method for the evaluation}

We evaluated angular resolution of gamma rays by the selected events. Fig 5 shows the schematic picture of angle measurement. We compared two angle. One is the angle reconstructed from momentum and emission angle of electron pair, and the other is the angle of a line which connecting interaction points.

\subsection{Result}

Fig $[$ shows the image of pointing result. Angle difference of $\mathrm{X}$ and $\mathrm{Y}$ projection are drawn in the $2 \mathrm{D}$ histogram. The bright center is the signal spot. We evaluated that the angular resolution is 0.7 deg. (@130-300 MeV) from the width of the spot. This result prove the emulsion telescope is superior than existing telescopes from the view point of angular resolution. 


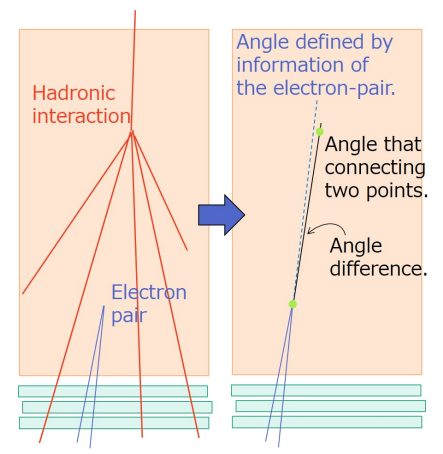

Figure 5: Conceptual scheme of evaluation of angular resolution.

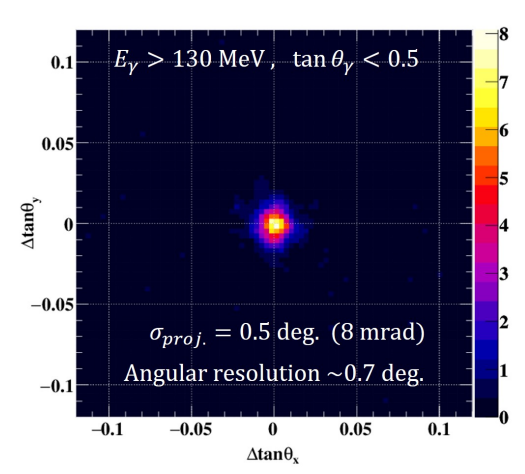

Figure 6: Image of gamma-ray pointing to vertexes of hadronic events.

\section{Conclusion}

We aim to improve angular resolution of gamma-ray telescope for gamma-ray astronomy. In 2015, we performed a balloon flight in Australia and analysis is undergoing. In this flight data, we selected gamma rays attaching to hadronic interactions and evaluate angular resolution by pointing accuracy to the vertex points. The result was $0.7 \mathrm{deg}$. (130 MeV $<E<300 \mathrm{MeV}$ ). This result indicates that emulsion telescope can realize significant improvement of quality of gamma-ray observation.

\section{References}

[1] F. ACERO, et. al., June 2015, FERMI LARGE AREA TELESCOPE THIRD SOURCE CATALOG, The Astrophysical Journal 218:23 (41pp)

[2] S. Takahashi, et. al., August 2010, Time stamp technique using a nuclear emulsion multi-stage shifter for gamma-ray telescope, Nucl. Inst. Meth. Phys., v 620, 2-3, 11-21, p 192-195

[3] S. Takahashi, et al., July 2016, GRAINE 2015, a balloon-borne emulsion $\gamma$-ray telescope experiment in Australia, Prog. Theor. Exp. Phys. 073F01 (12 pages)

[4] M. yoshimoto, et. al., TO BE PUBLISHED, Nuclear emulsion readout system HTS aimed at scanning area of a thousand square meters, Prog. Theor. Exp. Phys. 